\title{
Oxidative Stress in the Poultry Gut: Potential Challenges and Interventions
}

\author{
Birendra Mishra* and Rajesh Jha
}

Department of Human Nutrition, Food and Animal Sciences, College of Tropical Agriculture and Human Resources, University of Hawaii at Manoa, Honolulu, HI, United States

\section{OPEN ACCESS}

Edited by:

Pietro Celi,

DSM, United States

Reviewed by:

Maria Walsh,

DSM, Switzerland

Vincenzo Tufarelli,

Università degli Studi di Bari, Italy

*Correspondence:

Birendra Mishra

bmishra@hawaii.edu

Specialty section:

This article was submitted to

Animal Nutrition and Metabolism,

a section of the journal

Frontiers in Veterinary Science

Received: 17 October 2018

Accepted: 11 February 2019

Published: 04 March 2019

Citation:

Mishra B and Jha R (2019) Oxidative

Stress in the Poultry Gut: Potential

Challenges and Interventions.

Front. Vet. Sci. 6:60.

doi: 10.3389/fvets.2019.00060
The gastrointestinal tract (GIT) provides the biological environment for nutrient digestion and absorption, and protection from pathogens and toxins. Broilers are fast growing because of the great potential of intestinal epithelia for nutrient absorption, and efficient conversion of nutrient to muscle. Physiologically, reactive oxygen species (ROS) and reactive nitrogen species (RNS) are generated by GIT epithelial cells either from oxygen metabolism or by enteric commensal bacteria and regulate gut health. However, increased production of ROS elevates free radical production and antioxidant insults resulting in oxidative stress. Oxidative stress in poultry GIT is derived from nutritional, environmental heat stress, and pathological factors, which alters overall performance as well as meat and egg quality. Supplementation of exogenous vitamins, antioxidants, and plant extract having antioxidant properties scavenge ROS and are beneficial in mitigating oxidative stress in the GIT. This review highlights the involvement of oxidative stress in the gastrointestinal functionality of poultry and potential intervention strategies to maintain redox balance in the GIT.

\section{Keywords: oxidative stress, gastrointestinal tract, antioxidant, poultry production, heat stress}

\section{INTRODUCTION}

Poultry is one of the fastest growing animal industry and has a substantial contribution to food security and nutrition. Poultry meat and eggs are among the most common animal source of food consumed at the global level. The poultry and egg industries are among the largest agricultural commodities globally. Over the period, immense improvements have been made in genetics, feed conversion ratio, fat reduction, and breast size of broiler chickens and significant improvement in the hen-day egg production and egg quality in laying hen $(1,2)$. In the poultry industry, the feed is the major component of the total cost for meat and egg production. Also, feed exposes the birds to a wide variety of factors through the gastrointestinal tract (GIT), and affect poultry health and production.

The GIT is a highly complex and dynamic organ, which plays a critical role in nutrient absorption and immune response (3). Intestinal mucosa, a site for nutrient absorption, is composed of heterogeneous cell populations, epithelial cells, and connective tissues. The intestinal epithelia are exposed continuously to a wide variety of potentially harmful substances and act as a selective barrier between the tissues and luminal environment of the GIT. There are several stressors such as feed toxin; infectious agents induce the cellular free radicals' generation results in redox imbalance. This stress can negatively affect the delicate balance among the components of the chicken GIT, which in turn, affect the health status and productivity of poultry. The purpose of this review is to 
provide updated information on different oxidative stressors, to elucidate the impact of oxidative stress on the pathophysiology of poultry GIT and potential interventions to mitigate the effects.

\section{OXIDATIVE STRESS}

Stresses in commercial poultry result from environmental, nutritional, microbiological, and management factors which negatively impact poultry health and production. Oxidative stress is downstream of all these stresses. Oxidative stress in the cells/tissues results from an imbalance between free radical production and endogenous antioxidant defense and leads to lipid peroxidation, protein nitration, DNA damage, and apoptosis. Cells are exposed continuously with the free radicals generated during the physiological oxygen metabolism (4). Both reactive oxygen species (ROS) and reactive nitrogen species (RNS) at certain levels are signaling molecules involved in homeostasis. However, excessive production of ROS and RNS or their inefficient scavenging leads to oxidative stress. ROS, including superoxide, hydrogen peroxide, and the hydroxyl radical radicals, are generated by oxygen metabolism and further balanced by the rate of oxidant formation and the rate of oxidant elimination. The intracellular reduction of ROS is physiologically scavenged by superoxide dismutase, catalase, and glutathione peroxidase (GPX) (5). Superoxide dismutase (SOD1 and SOD2) catalyze the dismutation of the superoxide anion $\left(\mathrm{O}_{2}^{-}\right)$to $\mathrm{H}_{2} \mathrm{O}_{2}$ (6), which in turn, is decomposed into $\mathrm{H}_{2} \mathrm{O}$ and $\mathrm{O}_{2}$ by catalase, while GPX reduces lipid hydroperoxides by incorporating glutathione (6). The RNS that are by-products of nitric oxide synthases (NOS) are expressed in selected cells of the intestinal mucosa and submucosal regions. The NOS metabolizes arginine to citrulline and forms the nitric oxide radical $(\mathrm{NO} \bullet)$ which is crucial for cellular function including neurotransmission and immunomodulation. However, overproduction of nitric oxide radicals' damages intestinal mucous membrane and impaired nutrient utilization (7). Both ROS and RNS can contribute to lipid peroxidation especially cell membrane lipids and lipoproteins since they are rich in polyunsaturated fatty acids. The end product of lipid peroxidation is 4hydroxynonenal, which increases oxidative damage to the cell membrane and impair the cell signaling and mitochondrial dysfunctions. Inflammation in GIT is mediated through several stressors/infections which in turn generate ROS and disrupt redox balance.

\section{OXIDATIVE STRESS ASSOCIATED WITH ENVIRONMENTAL HEAT STRESS}

The high temperature is one of the most challenging environmental stressors associated with poultry production (8). Heat stress is a major source of systemic oxidative stress since it causes a redox imbalance between the pro- and anti-oxidants in favor of prooxidants. Heat stress has been shown to alter the feed intake, poor growth performance, immunosuppression, hypoxia, and high mortality $(9,10)$. Heat stress also deteriorates the meat quality of chicken (11). Birds under cyclic heat stress display less crypt depth, mucous area, and villus height of small intestine (12), leading to negative impact on nutrient absorption. Also, heat stress causes intestinal epithelial cell injury, and apoptosis contributes to intestinal hyperpermeability which causes the influx of bacterial products from the intestinal lumen into the circulatory system and affects organ systems. The ROS components such as superoxide anions, hydrogen peroxide, and hydroxyl radicals are produced in the mitochondria and act as signaling intermediates (5). Under physiological conditions, generated antioxidant enzymes rapidly eliminate ROS. Several studies link oxidative stress with heat stress or lipopolysaccharide (LPS), and suggest synergistic augmenting of cell death and increased ROS generation in specific cells (13). Heat stress also activates the chicken hypothalamus, pituitary adrenal axis, resulting in elevated serum corticosterone, which in turn decreases food intake, body weight gain, relative immune organ weight, and innate immunity. This neuroimmune dysfunction further alters intestinal-immune barrier, allowing pathogenic bacteria to migrate through the intestinal mucosa and generating an inflammatory infiltrate. Inflammation of the intestine also decreases nutrition absorption and consequently decrease in weight gain (14).

\section{OXIDATIVE STRESS ASSOCIATED WITH FEED TOXINS}

Poultry feeds/feed ingredients are often contaminated with a wide range of environmental toxicants, bacterial and fungal toxins, and known to affect the gut health. The intestinal luminal epithelial cells and the tight junction proteins between two adjacent epithelial cells from the barrier and thus preventing paracellular absorption of toxins. Oxidative stress alters not only the cellular processes but also the intestinal barrier function. Mycotoxins are metabolites produced in a strainspecific way by a wide range of fungi, particularly molds. The common mycotoxins are aflatoxin, zearalenone, deoxynivalenol, trichothecenes, fumonisin, T-2 toxin, and ochratoxin. Once these toxins come in contact with the epithelial cells or during the absorption, the GIT is greatly impacted by the induction of oxidative stress. It has been shown that the chronic, longterm exposure to even low levels of mycotoxins may impact the immune system and intestinal integrity and compromise the blood phagocytic activity in chickens (15). Trichothecenes are a group of mycotoxins (deoxynivalenol, T-2 toxin, and fumonisin B1) which are mainly produced by fungi of the genus Fusarium. These mycotoxins generate ROS which induces lipid peroxidation, alters the cellular redox signaling, antioxidant status, and membrane integrity of the cells. Trichothecenes also increase the intestinal epithelial permeability. Together, mycotoxins increase cellular apoptosis and affect poultry health and production.

Arsenic is widely distributed in water, food, and the environment. It is highly toxic and causes adverse effects 
on digestion and absorption of nutrients, resulting in potential losses to poultry growth. Heavy or chronic arsenic exposure induces lipid peroxidation, decreases antioxidants, and eventually trigger apoptosis in several body tissues of poultry (14). Copper, arsenic, and their combination induce the inflammation and the destruction of the intestinal mucosa (16).

Ammonia is one of the primary sources of the air contaminant in the poorly ventilated poultry house. High levels of ammonia decrease growth rate, body weight, and alter feed efficiency (17). Longer exposure to ammonia also causes several health issues and compromise the welfare of broilers (18). The absorption capacity of the intestine depends on the number and size of villi. Chicken exposed to high concentration of ammonia has much lower villus height and crypt depth among different segments of the small intestine. Ammonia also exerts negative impacts on immune organ development of chickens, which may cause enormous damages to nutrient absorption and immune system (18). It has also been reported that ammonia exposure increases the activity of creatine kinase and decreased activity of serum T-SOD producing oxidative stress and apoptosis of mucosal structure (19).

\section{OXIDATIVE STRESS ASSOCIATED WITH MICROORGANISMS}

In poultry production, intestinal health and function play a critical role in efficient feed utilization and growth, and the overall profitability of the farm. The GIT microbiota mainly consists of bacteria, fungi, and protozoa. Microbiota population varies across the compartment with maximal at the distal segments of the GIT (20). Intestinal epithelial in response to commensal bacteria generate ROS, which serves as a second messenger and participates in cellular signaling. Tight junctions between intestinal epithelial cells from the barrier and prevent the invasion of the microorganism (21, 22). Studies have suggested that interaction of mucosa with microbes or their toxin triggers oxidative stress. Coccidiosis is among the most common parasitic diseases of poultry. Eimeria primarily produces oxidative stress, thereby destroy the intestinal epithelial barrier and tight junctions, lipid peroxidation, antioxidants insult, as a result, infected birds display reduced feed intake, absorption of nutrients and decreases weight gains (23). Environmental heat stress affects the intestinal epithelial cells and further stimulate intestinal bacteria and bacterial LPS. The LPS is known to induce apoptosis and injury in various cell types (24).

\section{ANTI-OXIDATIVE SYSTEMS IN GIT}

The intestinal mucosa is responsible for the absorption of nutrient, and the antioxidant system maintains diverse microbiota in the luminal epithelia. The intestinal mucosa is directly exposed to both feed and non-feed substances. Above physiological level, production of ROS/RNS results in intestinal inflammation and impair the absorption capacity.
It has been reported that the broilers fed with the oxidized oils/fats imbalance antioxidants and immune response within the intestinal mucosa (25). As the first line of defense against oxidative stress, the intestinal mucosa contains an extensive antioxidants defense system including enzymes (CAT, SOD, or GPX) and non-enzymatic endo- and exo-genous scavengers like glutathione, transient ions (e.g., $\mathrm{Fe} 2^{+}, \mathrm{Cu}^{+}$) or flavonoids (26). Glutathione and SOD are intracellular antioxidants, widely distributed in the small intestine, and their abundances are at a higher level during intestinal development (27).

\section{MITIGATION OF OXIDATIVE STRESS}

The feeds intake, digestion, and subsequent absorption of nutrients in the intestine produce free radicals and imbalance antioxidant system in the intestinal mucosa resulting oxidative stress (28). Also, oxidative stress damages to the intestinal mucosa impede the efficient digestion and absorption of nutrients and adversely influences normal animal growth (29). Dietary inclusion of antioxidant compounds reduces intestinal free radicals, and also help in maintaining the intestinal mucosa. Several studies have suggested that oxidative stress predisposes the birds to various pathological and welfare situation. Therefore, it is essential to formulate a cost-effective strategy to mitigate oxidative stress. Supplementations of vitamin C and E, improve antioxidant ability and immune performance (30). Alpha lipoic acid, possesses both fat and water soluble, is a potent antioxidant and is protective against oxidative damages in the poultry intestine (31). The inclusion of polyphenol compounds also exhibits potent antioxidant activity (32). Equol which is derived from the isoflavonoid daidzein, a major isoflavone of soybean, can hinder oxidative modification induced by ROS (33). Equol protect protects intestinal epithelial cells from oxidative damage by promoting the expression of antioxidant genes, increasing the activities of antioxidant enzymes, and by enhancing antioxidant capacity (34). Dietary galacto-oligosaccharides, as a prebiotic, stabilizes intestinal integrity and prevent against oxidative damages (35). The supplementation of antioxidant-containing plants extracts such as Tulbaghia violacea is shown to have a beneficial effect on the rate of Eimeria oocyst shedding (23). Dietary supplementation of L-glutamine also prevents Necrotic enteritis in antibiotic-free diets (36). Dietary glutamate and Nacetylcysteine induce several antioxidant genes and inflammatory biomarkers in the intestinal mucosa which alleviate LPS-induced intestinal inflammation (37) and can be potentially be used in the chicken diet. Therefore, based on the stress, an individual ingredient or in combination can be used to mitigate oxidative stress in the GIT.

\section{CONCLUSION}

Oxidative stress in the poultry GIT is produced by the nutritional factors, environmental factors like heat stress, and pathological factors. These stresses have a negative impact on broiler growth and production as well as the quality of 
meat and egg produced. Currently, different antioxidants like exogenous vitamins, antioxidants, and plant extract are used individually or in combination to prevent the oxidative stress in poultry. These anti-oxidants scavenge the reactive oxygen species and reactive nitrogen species to mitigating oxidative stress in the GIT at varying level. Further studies are required to investigate the effects of antioxidants in different combination to mitigate oxidative stress in broiler chicken.

\section{REFERENCES}

1. Richards MP, Proszkowiec-Weglarz M. Mechanisms regulating feed intake, energy expenditure, and body weight in poultry. Poult Sci. (2007) 86:1478-90. doi: $10.1093 / \mathrm{ps} / 86.7 .1478$

2. Reyer H, Hawken R, Murani E, Ponsuksili S, Wimmers K. The genetics of feed conversion efficiency traits in a commercial broiler line. Sci Rep. (2015) 5:16387. doi: $10.1038 /$ srep 16387

3. Lan Y, Verstegen M, Tamminga S, Williams B. The role of the commensal gut microbial community in broiler chickens. Worlds Poult Sci J. (2005) 61:95-104. doi: 10.1079/WPS200445

4. Estevez M. Oxidative damage to poultry: from farm to fork. Poult Sci. (2015) 94:1368-78. doi: 10.3382/ps/pev094

5. Kurutas EB. The importance of antioxidants which play the role in cellular response against oxidative/nitrosative stress: current state. Nutr J. (2016) 15:71. doi: 10.1186/s12937-016-0186-5

6. Fukai T, Ushio-Fukai M. Superoxide dismutases: role in redox signaling, vascular function, and diseases. Antioxid Redox Signal. (2011) 15:1583-606. doi: 10.1089/ars.2011.3999

7. Sklyarov AY, Panasyuk NB, Fomenko IS. Role of nitric oxide-synthase and cyclooxygenase/lipooxygenase systems in development of experimental ulcerative colitis. J Physiol Pharmacol. (2011) 62:65-73.

8. Altan O, Pabuçcuoglu A, Altan A, Konyalioglu S, Bayraktar H. Effect of heat stress on oxidative stress, lipid peroxidation and some stress parameters in broilers. Br Poult Sci. (2003) 44:545-50. doi: 10.1080/00071660310001618334

9. Mujahid A, Akiba Y, Toyomizu M. Acute heat stress induces oxidative stress and decreases adaptation in young white leghorn cockerels by downregulation of avian uncoupling protein. Poult Sci. (2007) 86:364-71. doi: $10.1093 / \mathrm{ps} / 86.2 .364$

10. Yahav S, Goldfeld S, Plavnik I, Hurwitz S. Physiological responses of chickens and turkeys to relative humidity during exposure to high ambient temperature. J Therm Biol. (1995) 20:245-53. doi: 10.1016/0306-4565(94)00046-L

11. Hashizawa Y, Kubota M, Kadowaki M, Fujimura S. Effect of dietary vitamin $\mathrm{E}$ on broiler meat qualities, color, water-holding capacity and shear force value, under heat stress conditions. Anim Sci J. (2013) 84:732-6. doi: $10.1111 /$ asj.12079

12. Marchini CFP, Silva PL, Nascimento MRBM, Beletti ME, Silva NM, Guimaraes EC. Body weight, intestinal morphometry and cell proliferation of broiler chickens submitted to cyclic heat stress. Int J Poult Sci. (2011) 10:455-60. doi: 10.3923/ijps.2011.455.460

13. Quinteiro-Filho WM, Ribeiro A, Ferraz-de-Paula V, Pinheiro ML, Sakai M, Sá LR, et al. Heat stress impairs performance parameters, induces intestinal injury, and decreases macrophage activity in broiler chickens. Poult Sci. (2010) 89:1905-14. doi: 10.3382/ps.2010-00812

14. Zhao H, He Y, Li S, Sun X, Wang Y, Shao Y, et al. Subchronic arsenisminduced oxidative stress and inflammation contribute to apoptosis through mitochondrial and death receptor dependent pathways in chicken immune organs. Oncotarget. (2017) 8:40327-44. doi: 10.18632/oncotarget.16960

15. Wu Q, Dohnal V, Huang L, Kuca K, Yuan Z. Metabolic pathways of trichothecenes. Drug Metab Rev. (2010) 42:250-67. doi: 10.3109/03602530903125807

16. Wang Y, Zhao H, Liu J, Shao Y, Li J, Luo L, et al. Copper and arsenic-induced oxidative stress and immune imbalance are associated with activation of heat

\section{AUTHOR CONTRIBUTIONS}

$\mathrm{BM}$ reviewed the literature and drafted the manuscript. RJ reviewed the manuscript and provided critical review and suggestion and comments.

\section{FUNDING}

CTAHR, USDA/NIFA-Multistate Fund.

shock proteins in chicken intestines. Int Immunopharmacol. (2018) 60:64-75. doi: 10.1016/j.intimp.2018.04.038

17. Miles DM, Branton SL, Lott BD. Atmospheric ammonia is detrimental to the performance of modern commercial broilers. Poult Sci. (2004) 83:1650-4. doi: $10.1093 / \mathrm{ps} / 83.10 .1650$

18. Wei FX, Xu B, Hu XF, Li SY, Liu FZ, Sun QY, et al. The effect of ammonia and humidity in poultry houses on intestinal morphology and function of broilers. J Anim Vet Adv. (2012) 11:3641-46. doi: 10.3923/javaa.2012.3641.3646

19. Zhang J, Li C, Tang X, Lu Q, Sa R, Zhang H. Proteome changes in the small intestinal mucosa of broilers (Gallus gallus) induced by high concentrations of atmospheric ammonia. Proteome Sci. (2015) 13:9. doi: 10.1186/s12953-015-0067-4

20. Gabriel I, Lessire M, Mallet S, Guillot JF. Microflora of the digestive tract: critical factors and consequences for poultry. Worlds Poult Sci J. (2006) 62:499-511. doi: 10.1017/S0043933906001115

21. Anderson JM, Van Itallie CM. Tight junctions and the molecular basis for regulation of paracellular permeability. Am J Physiol. (1995) 269:467-75.

22. Ulluwishewa D, Anderson RC, McNabb WC, Moughan PJ, Wells JM, Roy NC. Regulation of tight junction permeability by intestinal bacteria and dietary components. J Nutr. (2011) 141:769-76. doi: 10.3945/jn.110.135657

23. Naidoo V, McGaw LJ, Bisschop SP, Duncan N, Eloff JN. The value of plant extracts with antioxidant activity in attenuating coccidiosis in broiler chickens. Vet Parasitol. (2008) 153:214-19. doi: 10.1016/j.vetpar.2008. 02.013

24. Guo S, Al-Sadi R, Said HM, Ma TY. Lipopolysaccharide causes an increase in intestinal tight junction permeability in vitro and in vivo by inducing enterocyte membrane expression and localization of TLR-4 and CD14. Am J Pathol. (2013) 182:375-87. doi: 10.1016/j.ajpath.2012.10.014

25. Liang F, Jiang S, Mo Y, Zhou G, Yang L. Consumption of oxidized soybean oil increased intestinal oxidative stress and affected intestinal immune variables in yellow-feathered broilers. Asian-Austr J Anim Sci. (2015) 28:1194-201. doi: 10.5713/ajas.14.0924

26. Circu ML, Aw TY. Intestinal redox biology and oxidative stress. Semin Cell Dev Biol. (2012) 23:729-37. doi: 10.1016/j.semcdb.2012.03.014

27. Tang D, Wu J, Jiao H, Wang X, Zhao J, Lin H. The development of antioxidant system in the intestinal tract of broiler chickens. Pouts Sci. (2019) 98:664-78. doi: $10.3382 / \mathrm{ps} / \mathrm{pey} 415$

28. Liu M, Gao R, Meng Q, Zhang Y, Bi C, Shan A. Toxic effects of maternal zearalenone exposure on intestinal oxidative stress, barrier function, immunological and morphological changes in rats. PLoS ONE. (2014) 9:e106412. doi: 10.1371/journal.pone.0106412

29. Yara S, Lavoie JC, Beaulieu JF, Delvin E, Amre D, Marci V, et al. Ironascorbate-mediated lipid peroxidation causes epigenetic changes in the antioxidant defense in intestinal epithelial cells: impact on inflammation. PLoS ONE. (2013) 8:e63456. doi: 10.1371/journal.pone.0063456

30. Min YN, Niu ZY, Sun TT, Wang ZP, Jiao PX, Zi BB, et al. Vitamin E and vitamin $\mathrm{C}$ supplementation improves antioxidant status and immune function in oxidative-stressed breeder roosters by up-regulating expression of GSH-Px gene. Poult Sci. (2018) 97:1238-44. doi: 10.3382/ps/pex417

31. El-Senousey HK, Chen B, Wang JY, Atta AM, Mohamed FR, Nie QH. Effects of dietary vitamin $\mathrm{C}$, vitamin $\mathrm{E}$, and alpha-lipoic acid supplementation on the antioxidant defense system and immune-related gene expression in broilers exposed to oxidative stress by dexamethasone. Poult Sci. (2018) 97:30-38. doi: $10.3382 / \mathrm{ps} / \mathrm{pex} 298$ 
32. Gerasopoulos K, Stagos D, Kokkas S, Petrotos K, Kantas D, Goulas P, et al. Feed supplemented with byproducts from olive oil mill wastewater processing increases antioxidant capacity in broiler chickens. Food Chem Toxicol. (2015) 82:42-9. doi: 10.1016/j.fct.2015.04.021

33. Liu HY, Zhang CQ, Zeng WD. Estrogenic and antioxidant effects of a phytoestrogen daidzein on ovarian germ cells in embryonic chickens. Domest Anim Endocrin. (2006) 31:258-68. doi: 10.1016/j.domaniend.2005.11.002

34. Lin X, Jiang S, Jiang Z, Zheng C, Gou Z. Effects of equol on H2O2-induced oxidative stress in primary chicken intestinal epithelial cells. Poult Sci. (2016) 95:1380-86. doi: 10.3382/ps/pew034

35. Varasteh S, Braber S, Akbari P, Garssen J, Fink-Gremmels J. Differences in susceptibility to heat stress along the chicken intestine and the protective effects of galacto-oligosaccharides. PLoS ONE. (2015) 10:9. doi: 10.1371/journal.pone.0138975

36. Xue GD, Barekatain R, Wu SB, Choct M, Swick RA. Dietary L-glutamine supplementation improves growth performance, gut morphology, and serum biochemical indices of broiler chickens during necrotic enteritis challenge. Poult Sci. (2018) 97:1334-41. doi: 10.3382/ps/pex444

37. Hou Y, Wang L, Yi D, Ding B, Yang Z, Li J, et al. N-acetylcysteine reduces inflammation in the small intestine by regulating redox, EGF and TLR4 signaling. Amino Acids. (2013) 45:513. doi: 10.1007/s00726-012-1295-x

Conflict of Interest Statement: The authors declare that the research was conducted in the absence of any commercial or financial relationships that could be construed as a potential conflict of interest.

Copyright (C) 2019 Mishra and Jha. This is an open-access article distributed under the terms of the Creative Commons Attribution License (CC BY). The use, distribution or reproduction in other forums is permitted, provided the original author(s) and the copyright owner(s) are credited and that the original publication in this journal is cited, in accordance with accepted academic practice. No use, distribution or reproduction is permitted which does not comply with these terms. 